\section{Alopecia neoplastica bei Mammakarzinom}

Zusammenfassung. Drei bzw. fünf Jahre nach der Diagnose und der Therapie von Mammakarzinomen entwickelten sich bei zwei Patientinnen umschriebene Alopezien am Kapillitium. Histopathologisch konnte in beiden Fällen eine kutane Metastasierung der Mammakarzinome nachgewiesen werden. Die Alopecia neoplastica ist ein seltener, klinisch morphologisch jedoch sehr charakteristischer Befund. Differenzialdiagnostisch abzugrenzen sind insbesondere die Alopecia areata und die verschiedenen Formen der narbigen Alopezien.

Neoplastic Alopecia Due to Breast Carcinoma - A Report on Two Cases. Three and five years, respectively, after diagnosis and treatment for breast carcinoma two female patients developed a circumscribed alopecia. Histopathologically this was due to the presence of metastatic breast carcinoma in both patients. Neoplastic alopecia is a rare but clinically and morphologically characteristic finding. The differential diagnosis comprises especially all forms of cicatrizing alopecia areata.

\section{Einleitung}

Typischerweise metastasieren viszerale Karzinome bevorzugt in die Leber, in die Lunge sowie in das jeweils dem Primärtumor anatomisch nachgeschaltete Lymphknotensystem. Kutane Metastasen viszeraler Karzinome werden in der Praxis hingegen wesentlich seltener beobachtet, wobei die in der Literatur angegebenen Häufigkeitsraten mit 0,7 - 9,0\% deutliche Unterschiede aufweisen $[16,17]$. Anhand der Datenlage größerer Statistiken konnte bei einer Gruppe von über 17000 Patienten mit viszeralen Karzinomen eine Häufigkeit kutaner Metastasen von 5,5\% berechnet werden [12]. Von besonderer Bedeutung für die betroffenen Patienten ist die Erkenntnis, dass bei $10-25 \%$ der Fälle eine kutane Metastase das erste Symptom eines bis dato nicht bekannten viszeralen Karzinoms darstellt [7]. Bei der diagnostischen Abklärung einer kutanen Karzinommetastase können geschlechtsspezifische Unterschiede die Suche nach einem möglicherweise noch unbekannten Primärtumor erleichtern. Während bei Männern Karzinome der

Akt Dermatol 2001; 27: 245-247

(c) Georg Thieme Verlag Stuttgart · New York ISSN 0340-2541

\author{
G. Wagner \\ Hautklinik Zentralkrankenhaus Bremerhaven \\ (Chefarzt: Dr. G. Wagner)
}

Lunge, des Ingestionstraktes oder des Urogenitalsystems gehäuft kutane Metastasen zeigen, finden sich bei Frauen überwiegend Karzinome der Mamma, deutlich seltener solche des Kolons, der Ovarien oder der Lunge [3]. Dabei ist das Mammakarzinom von allen viszeralen Karzinomen des Menschen mit Abstand am häufigsten der Ausgangspunkt einer kutanen Metastasierung. Neben der Lymphangiosis carcinomatosa ist auch die Alopecia neoplastica eine typische Metastasenform des Mammakarzinoms, was durch die nachfolgenden Kasuistiken gezeigt werden soll.

\section{Kasuistik 1}

Patientin: G. K., 62 Jahre, Hausfrau

\section{Anamnese}

1997 wurde bei der Patientin ein invasives tubuläres Karzinom der rechten Mamma operativ entfernt. Bei der gleichzeitig durchgeführten Lymphadenektomie fanden sich vier Lymphknotenmetastasen, während eine weitergehende Metastasierung zunächst ausgeschlossen werden konnte. Postoperativ erfolgte eine adjuvante Chemotherapie mit Cyclophosphamid und Epirubicin sowie eine prophylaktische Radiatio. Drei Jahre später entwickelte sich innerhalb weniger Wochen ein umschriebener, von deutlichen Schmerzen begleiteter Haarverlust am Kapillitium.

\section{Dermatologischer Befund}

Parietal rechts $1,5 \mathrm{~cm}$ durchmessender, teilweise eingesunkener, teilweise flach erhabener Alopezieherd mit zart rosa gefärbter, glasig wirkender Oberfläche. Zahlreiche Teleangiektasien, Haarfollikelostien nicht erkennbar. Palpatorisch derbe Konsistenz der in der Tiefe plattenartig tastbaren Strukturen (Abb. 1).

\section{Befunde diagnostischer Untersuchungen}

\section{Histopathologischer Befund}

Bei regelrechter Epidermis diffuse Infiltration im Korium durch teils solide tubuläre atypische Epithelkomplexe mit einer mäßiggradigen Zell- und Kernpolymorphie. Invasives Wachstum. Hormoneller Rezeptorstatus jeweils 70\% positiv für Östrogen- und Progesteronrezeptoren. 


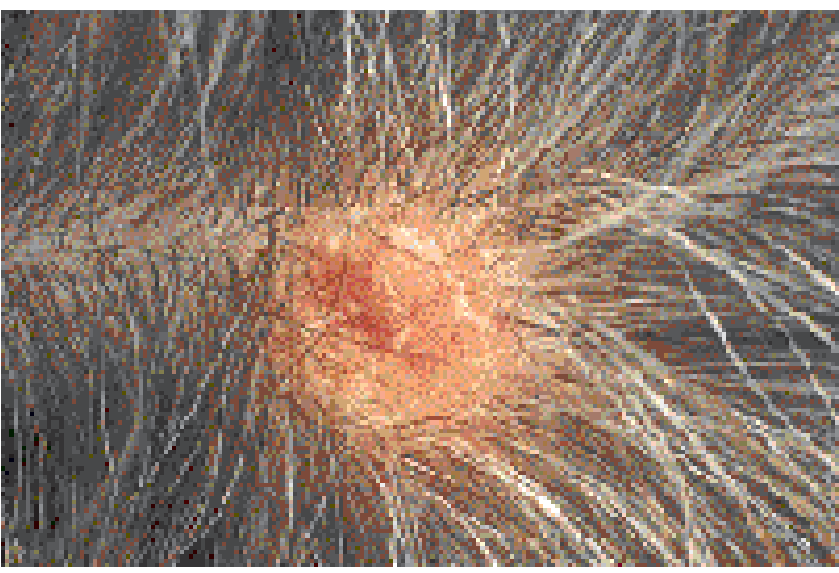

Abb.1 Alopecia neoplastica: Skleroatrophische Metastase.

Diagnose: Kutane Metastase eines mäßig differenzierten tubulären Adenokarzinoms. Das histopathologische Bild entspricht der Metastase eines Mammakarzinoms.

\section{Ergänzende Untersuchungen}

Bei den Staging-Untersuchungen konnte eine multilokuläre ossäre und intrahepatische Metastasierung nachgewiesen werden. Hinweise für ein möglicherweise unbekanntes Zweitkarzinom fanden sich nicht.

\section{Therapie und Verlauf}

Therapeutisch erfolgte eine Kondylen- und Inframedullar-Plattenosteosynthese Trochanter major links sowie eine Chemotherapie mit Mitoxantron.

\section{Kasuistik 2}

Patientin: K. T., 47 Jahre, Sekretärin

\section{Anamnese}

1995 wurde bei der Patientin die Diagnose eines invasiven tubulären Mammakarzinoms links gestellt. Nach brusterhaltender Operation und histopathologischem Ausschluss axillärer Lymphknotenmetastasen erfolgte eine Radiatio der linken Mamma. Die zum Zeitpunkt der Operation und in der Nachsorgeüberwachung durchgeführten Staging-Untersuchungen ergaben keine Hinweise für eine generalisierte Metastasierung. Im Frühjahr 2000 bemerkte die Patientin erstmals kleine Alopezieherde am behaarten Kopf, die in den folgenden sechs Monaten langsam an Größe zunahmen.

\section{Dermatologischer Befund}

Okzipital und parietal beidseits insgesamt vier bis zu $1 \mathrm{~cm}$ durchmessende, gelblich bräunlich tingierte, ganz flach erhabene Alopezieherde. Vollständiger Verlust der Haarfollikelostien, Teleangiektasien, derbe Konsistenz (Abb. 2).

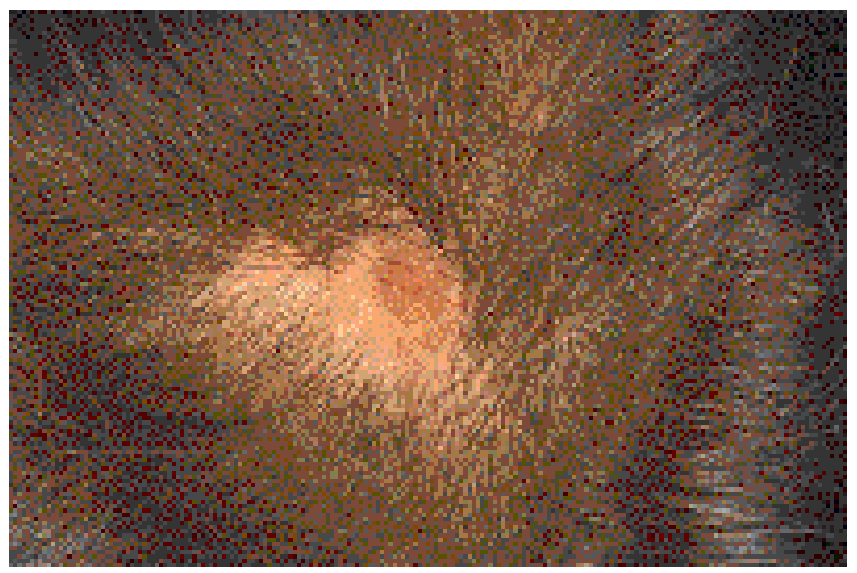

Abb. 2 Alopecia neoplastica: Plateauartig aufgeworfene Metastase.

\section{Befunde diagnostischer Untersuchungen}

Histopathologischer Befund

Bei unauffälliger Epidermis subepidermal eine diffuse Infiltration durch teils tubuläre, teils solide Epithelkomplexe mit einer deutlichen Zell- und Kernpolymorphie. Invasives Wachstum. Hormoneller Rezeptorstatus jeweils $90 \%$ Positivität für Östrogen- und Progesteronrezeptoren.

Diagnose: Hautmetastase eines wenig differenzierten tubulären Karzinoms. Der histopathologische Befund ist gut mit der Metastase eines Mammakarzinoms vereinbar.

\section{Ergänzende Untersuchungen}

Als Ergebnis der Staging-Untersuchungen musste eine diffuse Knochemarkmetastasierung festgestellt werden. Ein Zweitkarzinom konnte auch bei dieser Patientin nicht nachgewiesen werden.

\section{Therapie und Verlauf}

Als Behandlungsversuch wurde eine kombinierte Hormonund Zytostatikatherapie mit Goserelin und Mitoxantron eingeleitet.

\section{Diskussion}

In den westlichen Industrienationen ist das Mammakarzinom die häufigste maligne Tumorerkrankung der Frau. Bei seit Jahren global zunehmenden Inzidenzraten rechnet man in Deutschland heute mit 46000-56000 Neuerkrankungen pro Jahr [14]. Bei einer angegebenen Heilungsrate von 35-40\% kommt es somit bei mehr als der Hälfte der Patientinnen im Laufe der Erkrankung zur klinisch manifesten Generalisation [15].

In Abhängigkeit vom Tumordurchmesser zeigen sich häufig bereits bei der primären chirurgischen Versorgung der Patientinnen ipsilateral axilläre Lymphknotenmetastasen. Bei einem Tumordurchmesser von 6-10 mm wird der axilläre Lymphknotenbefall mit $10-30 \%$ angegeben, während bei einem Tumordurchmesser von über $20 \mathrm{~mm}$ in $60-80 \%$ der Fälle mit 
axillären Lymphknotenmetastasen gerechnet werden muss [15]. Neben der lymphogenen Ausbreitung kann auch die ebenfalls häufige hämatogene Metastasierung frühzeitig einsetzen. Bevorzugte Organsysteme der Metastasierung sind dabei das Skelettsystem, die Lunge, die Leber und das Gehirn. Aber auch kutane Metastasen sind keineswegs selten. Bei der Auswertung der Autopsiebefunde von 100 Patientinnen mit generalisiert metastasierten Mammakarzinomen fand sich bei 30\% der Fälle auch eine kutane Metastasierung [11]. Ein weiterer Aspekt, der die Bedeutung des Mammakarzinoms für die Dermatologie verdeutlicht, ist die zahlenmäßige Häufigkeit des Mammakarzinoms als Ursache einer kutanen Metastase im Vergleich zu allen anderen viszeralen Karzinomen. So fanden Brownstein und Helwig anhand klinischer und histopathologischer Befunde bei 242 Patientinnen mit kutanen Metastasen 168 Mammakarzinome, entsprechend einer Häufigkeit von 69\%. Erst mit weitem Abstand war das Kolonkarzinom der zweithäufigste Tumor innerhalb der untersuchten Gruppe, an dem im Vergleich zum Mammakarzinom nur 22 Patientinnen bzw. 9\% des Gesamtkollektivs erkrankt waren [3]. Bei vergleichbaren Untersuchungen von Post und Jänner konnten bei einer kleineren Gruppe von 63 Patientinnen 45 Mammakarzinome als Ausgangspunkt der kutanen Metastasierung nachgewiesen werden [13]. Ehlers und Krause fanden 20 Mammakarzinome innerhalb einer Gruppe von 26 kutan metastasierten Tumorpatientinnen [9]. Die häufigste Lokalisation kutaner Mammakarzinommetastasen sind das unmittelbare Op-Gebiet sowie die ventralen und lateralen Thoraxregionen. Die 5-Jahres-Rate dieser als Lokalrezidive verstandenen Metastasen beträgt je nach primärem Therapieverfahren 5$10 \%$ [5]. Betrachtet man bei metastasierten Patientinnen die lokalisatorische Verteilung der kutanen Metastasen am gesamten Integument, so finden sich diese bei $60-75 \%$ der betroffenen Patientinnen im Bereich der jeweiligen Thoraxregion des Mammakarzinoms [4,9]. Diese auffällige Häufigkeit der kutanen Metastasen wird durch die Anatomie der Lymphgefäße erklärbar, die ihren Ursprung im Bindegewebe der Milchdrüsenläppchen nehmen und im Bereich der Areola mit den dermalen Lymphkapillaren in Verbindung treten, die ihrerseits wiederum in die kutanen Lymphbahnen der Brustwand übergehen. Klinisch morphologisch manifestieren sich die Metastasen entweder in Form knotiger Strukturen oder als Lymphangiosis carcinomatosa vom Typ des Carcinoma erysipelatoides oder vom Typ des Cancer en cuirasse [2].

Eine seltene, jedoch überaus typische Variante der kutanen Mammakarzinommetastasen stellt die Alopecia neoplastica dar. Eine entsprechende Metastasierungsform beobachteten Brownstein und Helwig nur bei fünf ihrer 168 Patientinnen [3]. Post und Jänner konnten bei ihren 45 Patientinnen in sechs Fällen eine Beteiligung des Kapillitiums nachweisen [13]. Das klinische Bild der Alopecia neoplastica ist gekennzeichnet durch einen meist kreisrunden Haarausfall von bis zu mehreren Zentimetern Durchmesser, der in Form einzelner oder multipler Herde auftreten kann. Die Kopfhaut in den betroffenen Arealen wird entweder als skleroatrophisch oder als pseudopeladeartig beschrieben $[7,10]$. Die Haut ist typischerweise leicht eingesunken oder flach erhaben infiltriert von gelblichbräunlich bis rötlicher Färbung. Die Oberfläche wirkt glasig, teilweise mit diskreter Schuppung oder umschriebenen Krusten. Follikelostien sind nicht erkennbar. Der Palpationsbefund ist derb, vereinzelt auch in Form plattenartiger Strukturen, die sich in die Tiefe der Haut fortsetzen $[1,6,8]$.
Die Differenzialdiagnose der Alopecia neoplastica umfasst die Alopecia areata sowie die verschiedenen Formen der narbigen Alopezien einschließlich der sklerodermiformen Basalzellkarzinome und der singulären Herde eines chronisch diskoiden Lupus erythematodes am Kapillitium [10]. Bei flüchtiger Betrachtung des Befundes einer Alopecia neoplastica kann das Bild des kreisrunden Haarausfalls zur Fehldiagnose einer Alopecia areata verleiten [6]. Die eigentliche kutane Morphologie der Alopecia neoplastica ist jedoch so typisch, dass eine Alopecia areata meist bereits klinisch sicher ausgeschlossen werden kann. In allen Zweifelsfällen sollte insbesondere bei Patientinnen mit bekanntem Mammakarzinom eine histopathologische Abklärung erfolgen.

\section{Literatur}

${ }^{1}$ Baran R. Les métastases alopéciantes scléro-atrophiques des cancers mammaires. Dermatologica 1969; 138: 169-181

2 Bork K. Haut und Brust. 1. Aufl. Stuttgart: Fischer, 1995

${ }^{3}$ Brownstein MH, Helwig EB. Metastatic tumors of the skin. Cancer 1972; 29: $1298-1307$

${ }^{4}$ Brownstein MH, Helwig EB. Patterns of cutaneous metastasis. Arch Derm 1972; 105: 862-868

${ }^{5}$ Bubb C, Baumeister R, Bayerl A. Behandlung des lokalen und regionären Tumorrezidivs. In: Tumorzentrum München (Hrsg). Mammakarzinome. München: Eigendruck, 1998

${ }^{6}$ Cohen I, Levy E, Schreiber H. Alopecia neoplastica due to breast carcinoma. Arch Derm 1961; 84: 490-492

${ }^{7}$ Delacretaz J, Perroud H. Metastatische Karzinome der Haut. In: Korting GW (Hrsg). Dermatologie in Praxis und Klinik. Stuttgart: Thieme, 1981

${ }^{8}$ Domonkos AN. Alopecia neoplastica. Arch Derm 1972; 105: 120 122

${ }^{9}$ Ehlers G, Krause W. Über kutane Metastasen maligner Tumoren innerer Organe. Hautarzt 1970; 21: 66-75

${ }^{10}$ Goerz G, Kind R. Narbige Alopezien. In: Orfanos CE (Hrsg). Haar und Haarkrankheiten. Stuttgart: Gustav Fischer, 1979

${ }^{11}$ Harris JR, Morrow M, Bonadona G. Cancer of the breast. In: De Vita VT, Hellmann S, Rosenberg SA (Hrsg). Cancer Principles and Practice of Onkology. Philadelphia: Lippincott, 1993

${ }^{12}$ Marghescu S. Hautmetastasen interner Karzinome. Eine kritische Literaturstudie. H u G 1989; 64: 805- 809

${ }^{13}$ Post B, Jänner M. Zur Häufigkeit und Lokalisation des sekundären Hautkarzinoms. Hautarzt 1974; 25: 17-20

${ }^{14}$ Sauer H, De Waal JC, Hölzel D. Nachsorge. In: Tumorzentrum München (Hrsg). Mammakarzinome. München: Eigendruck, 1998

${ }^{15}$ Schmidt-Matthiesen H, Bastert G. Gynäkologische Onkologie. 4. Aufl. Stuttgart: Schattauer, 1993

${ }^{16}$ Spencer PS, Helm TN. Skin metastases in cancer patients. Cutis 1987; 39: 119-121

${ }^{17}$ Winer LH, Wright ET. Über den sekundären (metastatischen) Hautkrebs. Hautarzt 1960; 11: 23-27

\section{Dr. G. Wagner}

Hautklinik Zentralkrankenhaus

Reinkenheide

Postbrookstraße 103

27574 Bremerhaven 